\title{
A priori error estimation of magnetic material characteristics using stochastic uncertainty analysis
}

\author{
Ahmed Abou-Elyazied Abdallh, Guillaume Crevecoeur, and Luc Dupré \\ Department of Electrical Energy, Systems and Automation, Ghent University, Belgium \\ 41Sint-Pietersnieuwstraat, Ghent B-9000, Belgium \\ Ahmed.Abdallh@UGent.be
}

\begin{abstract}
By interpreting electromagnetic or mechanical measurements with a numerical model of the considered electromagnetic device, magnetic properties of the magnetic circuit of that device can be estimated by solving an inverse numerical electromagnetic problem. Due to measurement noise and uncertainties in the forward model, errors are made in the reconstruction of the material properties. This paper describes the formulation and implementation of the error estimation and the prediction of which measurements that need to be carried out for accurate magnetic material characterization. Stochastic uncertainty analysis, based on Cramér-Rao bound (CRB), is introduced and applied to the magnetic material characterization of a Switched Reluctance Motor (SRM) starting from mechanical (torque) and local magnetic measurements. The traditional CRB method that estimates the error due to measurement noise is extended with the incorporation of stochastic uncertain geometrical model parameters.
\end{abstract}

\section{Introduction And Methodology}

A Switched Reluctance Motor (SRM) is an electromagnetic device (EMD), which is widely used, nowadays, in industry. In order to precisely predict the machine performance, its magnetic $B-H$ characteristics need to be known. The application of Epstein or single sheet tester measurements on a separate sheet may result in an inaccurate approach for performance prediction. Indeed, manufacturing processes may alter significantly the material characteristics, see e.g. [1]. Therefore, the identification of the magnetic material properties after construction of the EMD is a more accurate approach. This identification procedure can be implemented by solving an inverse approach, based on a coupled numerical-experimental procedure, [2].

This inverse procedure has a resolution, which is difficult to determine due to measurement noise and uncertainties of the geometrical parameters in the EMD forward model $\mathbf{f}$. This model $\mathbf{f}$ has as input the magnetic material parameters $\mathbf{x} \in$ $\mathbb{R}^{q \times 1}$ and uncertain geometrical parameters $\mathbf{u} \in \mathbb{R}^{p \times 1}$, and wants to simulate the measurements $\mathbf{y} \in \mathbb{R}^{m \times 1}: \mathbf{y}=\mathbf{f}(\mathbf{x}, \mathbf{u})+$ $\mathbf{n}$, where $\mathbf{n}$ represents the measurement noise.

In this paper, we use 4 different inverse approaches for the magnetic material characterization $(q=3$, see [2]) of a 6/4 SRM (Fig. 1a): static torque measurements for different rotor angles $(m=19)$ and three local magnetic induction measurements carried out respectively at a stator yoke, a rotor yoke and at a rotor pole. We investigate in detail the influence of $p=5$ different uncertain geometrical parameters $\mathbf{u}$ : air gap $(\delta)$, stator yoke (tooth width $t_{s p}$, inner diameter $D_{s i}$ ) and rotor yoke (tooth width $t_{r p}$, external diameter $D_{r e}$ ) dimension values in the motor model on the accuracy of the results of 4 different inverse approaches.

We propose the use of the stochastic Cramér-Rao bound (CRB) method, for quantifying the possible errors on recovered magnetic material parameters $\mathbf{x}$, introduced by $\mathbf{n}$ and $\mathbf{u}$.
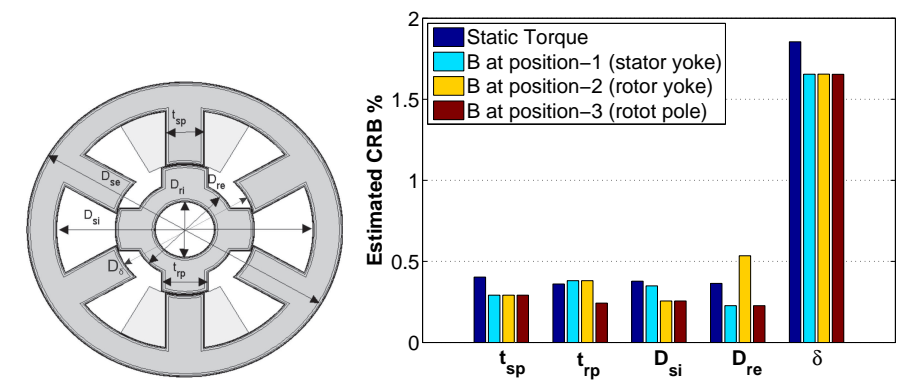

Fig. 1: (a) The schematic diagram of the $6 / 4$ SRM. $\delta$ is the air gap at alignment condition. (b) The estimated CRB due to the measurement noise and 5 different geometrical uncertainties of the studied SRM.

CRB is widely-used in signal processing [3] that calculates the lower bound on the variance of any unbiased estimator $\phi=\left[\mathbf{x}, \nu_{n}\right]$. We assume here that the measurements are Gaussian white noise with variance $\nu_{n}$. This CRB method can be extended when dealing with probabilistic model parameters $\mathbf{u}$, see [4] with estimator $\psi=\left[\mathbf{x}, \mathbf{u}, \nu_{n}\right]$. We assume that the random $\mathbf{u}$ is Gaussian prior with mean $\mu_{u}$ and variance $\nu_{u}$. Based on the estimated error, we are able to determine which measurements to use in the inverse procedure. In this way, the inverse problem is solved with high resolution.

\section{RESUlTS AND DISCUSSION}

Fig. 1b illustrates the estimated CRB values due to measurement noise and uncertainty in the 5 geometrical parameter values of the SRM. From the experimental results, it is observed that noise in the static torque measurements is higher than the noise in the magnetic measurements. Here we assume $\nu_{n, \text { Torque }}=6 \nu_{n, B}=4 e^{-6} \cdot \mu_{u}$ are the measured geometrical values with $\nu_{u}=10^{-6}$.

It can be observed from Fig. 1b that the accuracy of the inverse approach highly depends on the definition of the inverse problem (mechanical or magnetic measurements) and the considered uncertain parameter. For example, the uncertainty in the air gap thickness value gives the worse results for inverse problems based on mechanical measurements, and inverse problems based on local magnetic measurements are generally more accurate than the ones based on mechanical measurements. The complete algorithm, the experimental results and the complete analysis will be discussed in detail, in the full paper.

\section{REFERENCES}

[1] M. Takezawa, et. al., IEEE Trans. on Magnetics, vol. 42, pp. 2790-2792, 2006.

[2] A. Abdallh, et. al., IEEE Trans. Magn., vol. 52, pp. 4157-4160, 2009.

[3] C. Muravchik, et. al., IEEE Trans. Sign. Proc., vol. 49, pp. 470-484, 2001

[4] B. Radich, et. al., IEEE Trans. Biom. Eng., vol. 42, pp. 233-241, 1995. 